\title{
Post-transplantation outcomes of sensitized mechanical circulatory support patients
}

\author{
Jong-Chan Youn ${ }^{1}$, Jin-Jin Kim¹, Sang Hong Baek', Jon Kobashigawa² \\ 1Department of Internal Medicine-Cardiology, The Catholic University of Korea, Seoul St. Mary's Hospital, Seoul, Korea \\ ${ }^{2}$ Department of Cardiology, Cedars-Sinai Medical Center, Los Angeles, CA, USA
}

Background: Sensitization, defined as the presence of circulating antibodies, presents challenges, particularly in patients undergoing heart transplantation $(\mathrm{HTx})$ bridged with durable mechanical circulatory support (MCS). We aimed to investigate the post-transplantation outcomes of sensitized MCS patients.

Methods: Among 889 consecutively enrolled HTx recipients between 2010 and 2018 in Cedars-Sinai Medical Center, 86 sensitized MCS patients $(9.7 \%$, group A) were compared with sensitized non-MCS patients (group B, $n=189$ ), non-sensitized MCS patients (group C, $n=162$ ), and non-sensitized non-MCS patients (group $D, n=452$ ) regarding post-HTx outcomes, including the incidence of primary graft dysfunction (PGD), 1-year survival, and 1-year freedom from antibody-mediated rejection (AMR).

Results: Sensitized MCS patients (group A) showed comparable rates of PGD, 1-year survival, and 1-year freedom from AMR with groups $C$ and D. However, group A showed significantly higher rates of 1 -year freedom from AMR $(95.3 \%$ vs. $85.7 \%, P=0.02)$ and an earlier decline in panel-reactive antibody (PRA) levels $(P<0.01)$ than sensitized non-MCS patients (group $B)$. Desensitization therapy effectively reduced the levels of PRA in both groups $A$ and $B$. When group A was further divided according to the presence of preformed donor-specific antibodies (DSA), patients with preformed DSA showed significantly lower rates of 1-year freedom from AMR than those without $(84.2 \%$ vs. $98.5 \%, P=0.01)$.

Conclusions: Sensitized MCS patients showed significantly lower rates of AMR and an earlier decline in PRA levels following HTX than sensitized non-MCS patients. Removal of MCS at the time of transplantation might underlie these observations.

Corresponding author: Jong-Chan Youn

E-mail: jong.chan.youn@gmail.com

\footnotetext{
(c) The Korean Society for Transplantation

This is an Open Access article distributed under the terms of the Creative Commons Attribution Non-Commercial License (http://creativecommons.org/licenses/by-nc/4.0/) which permits unrestricted non-commercial use, distribution, and reproduction in any medium, provided the original work is properly cited.
} 